\title{
A TOTALLY ORDERED BAIRE SPACE FOR WHICH BLUMBERG'S THEOREM FAILS
}

\author{
RONNIE LEVY
}

Abstract. An elementary example of a totally ordered nonBlumberg Baire space is given.

A topological space $X$ is called Blumberg if for each $f: X \rightarrow \boldsymbol{R}$, there is a dense subset $D$ of $X$ such that $f \mid D$ is continuous. Bradford and Goffman proved in [2] that a metric space $X$ is Blumberg if and only if it is Baire. This result generalized the result of Blumberg in [1] that $\boldsymbol{R}$ is Blumberg. We give here an example of a totally ordered Baire space which fails to be Blumberg.

A totally ordered set $T$ is called an $\eta_{1}$-set if for any countable subsets $A$ and $B$ such that $a<b$ for each $a \in A$ and $b \in B$, there is an $x \in T$ such that $a<x<b$ for each $a \in A, b \in B$. Let $X$ be an $\eta_{1}$-set such that $|X|=2^{N_{0}}$ supplied with the order topology. (Such an $\eta_{1}$-set exists. See [3, p. 187].) Then $X$ is a Baire space. For suppose $\left\{G_{i}\right\}_{i=1}^{\infty}$ is a collection of open dense sets, and $(a, b)$ is a nonempty basic open set in $X$. We claim $(a, b) \bigcap_{i=1}^{\infty} G_{i} \neq \varnothing$. There is a nonempty open interval $\left(a_{1}, b_{1}\right) \subseteq(a, b) \cap G_{1}$. Inductively, we find nonempty open intervals $\left(a_{n}, b_{n}\right)$ such that $\left(a_{n+1}\right.$, $\left.b_{n+1}\right) \subseteq\left(a_{n}, b_{n}\right) \cap G_{n+1}$. Then $a_{i}<b_{j}$ for all $i, j$. Therefore, there is an $x \in X$ such that $\left\{a_{i}\right\}_{i=1}^{\infty}<x<\left\{b_{i}\right\}_{i=1}^{\infty}$. But then $x \in(a, b) \cap \bigcap_{i=1}^{\infty} G_{i}$. This proves that $X$ is Baire.

$X$ is a $P$-space without isolated points (see [3, Problem 13.P]). Hence any dense subset of $X$ is a $P$-space without isolated points. Therefore, if $f: X \rightarrow \boldsymbol{R}$ is a one-to-one function and $D$ a dense subset of $X$, then $f \mid D$ fails to be continuous at each point of $D$. Thus, $X$ fails to be Blumberg.

\section{REFERENCES}

1. H. Blumberg, New properties of all real functions, Trans. Amer. Math. Soc. 24 (1922), 113-128.

2. J. C. Bradford and C. Goffman, Metric spaces in which Blumberg's theorem holds, Proc. Amer. Math. Soc. 11 (1960), 667-670. MR 26 \#3832.

3. L. Gillman and M. Jerison, Rings of continuous functions, University Series in Higher Math., Van Nostrand, Princeton, N.J., 1960. MR 22 \#6994.

Department of Mathematics, Washington University, St. Louis, Missouri 63130

Received by the editors February 2, 1973.

AMS (MOS) subject classifications (1970). Primary 54C30, 54F05.

Key words and phrases. Baire space, Blumberg space, $P$-space, $\eta_{1}$-set.

(c) American Mathematical Society 1973 